\title{
Gravidez do Primeiro Filho: Papéis Sexuais, Ajustamento Conjugal e Emocional ${ }^{1}$
}

\author{
José Augusto Evangelho Hernandez ${ }^{2}$ \\ Instituição Educacional São Judas Tadeu \\ Universidade Federal do Rio Grande do Sul \\ Cláudio Simon Hutz \\ Universidade Federal do Rio Grande do Sul
}

\begin{abstract}
RESUMO - Esta investigação examinou as relações entre papéis sexuais, ajustamento conjugal e emocional de mulheres no período de gravidez da transição para a parentalidade. Participaram 135 mulheres recrutadas na rede pública de saúde (SUS). As gestantes estavam esperando os seus primeiros filhos e coabitavam com seus parceiros, independentemente de serem formalmente casadas. Os instrumentos utilizados foram o Bem Sex-Role Inventory (Bem, 1974), a Dyadic Adjustment Scale (Spanier, 1976) e a Escala Fatorial de Neuroticismo (Hutz \& Nunes, 2001). A coleta foi individual e, em geral, os instrumentos foram preenchidos na presença do entrevistador. Um número pequeno de questionários foi respondido no domicílio dos sujeitos e, posteriormente, devolvido aos pesquisadores. Os resultados mostraram relações significativas entre Papéis Sexuais e Ajustamento Conjugal. Os dados são discutidos à luz da Teoria de Esquema de Gênero e do conhecimento produzido na linha de pesquisa da transição para a parentalidade.
\end{abstract}

Palavras-chave: gravidez; papéis sexuais; transição para a parentalidade; ajustamento conjugal; ajustamento emocional/ neuroticismo.

\section{First Child Pregnancy: Sex Roles, Marital and Emotional Adjustment}

\begin{abstract}
The present investigation examined the relationship between sexual roles and pregnant women marital and emotional adjustment on the transition to parenthood. A hundred thirty three women participants were recruited from the public health care network in southern Brazil. The participants lived together with their partners and they were waiting for their first son or daughter. The tests used were the Bem Sex-Role Inventory (Bem, 1974), the Dyadic Adjustment Scale (Spanier, 1976) and the Neuroticism Factor Scale (Hutz \& Nunes, 2001). The data collection was individual and, in several cases, they fill the instruments in an interviewer presence. In a small amount of cases, participants responded to the instruments at home and later they returned them to the researcher. The results showed significant relationships between sex roles and marital adjustment. The results are discussed based on gender theories and on the international research on the transition to parenthood results.
\end{abstract}

Key words: pregnancy; sex roles; transition to parenthood; marital adjustment; emotional adjustment/neuroticism.

Esta pesquisa verificou as relações de papéis sexuais com o ajustamento conjugal e emocional em mães envolvidas com a gestação dos seus primeiros filhos. Este estágio da transição para a parentalidade é um período do ciclo vital humano caracterizado pelo início de mudanças fortemente impactantes sobre a vida das mulheres que o experimentam (Carter \& McGoldrick, 2001; Goldberg, Michaels \& Lamb, 1985; Knauth, 2001) e, portanto, se constitui num contexto estimulante para testar o modelo proposto pela psicóloga norte-americana Sandra Bem $(1974,1975,1977,1981)$ em sua Teoria do Esquema de Gênero.

Öhman, Grunewald e Waldenström (2003) apresentaram a gravidez como um período caracterizado por mudanças biológicas, psicológicas e sociais. A primeira gravidez é uma transição semelhante à menarca e à menopausa, uma passagem de um estágio de desenvolvimento para outro, com implicações na auto-imagem, nos valores, nos compor-

1 Esta pesquisa foi parcialmente financiada pelo Hospital de Clínicas de Porto Alegre/GPPG/Comissão Científica e Comissão de Pesquisa e Ética em Saúde com recursos financeiros do FIPE.

2 Endereço: Rua Dr. Ernesto Di Primio Beck 240, Bairro Partenon. Porto Alegre, RS. Brasil 91510-490.E-mail: hernandz@portoweb.com.br tamentos e nos relacionamentos interpessoais das mulheres. O primeiro trimestre da gravidez é um período de fusão no qual a mãe percebe o feto como uma parte fisicamente integrada de si mesma. No período seguinte, caracterizado pela diferenciação, o feto cresce como um indivíduo separado. A terceira fase é caracterizada pela separação psicológica e pelo crescimento da curiosidade das mulheres acerca do encontro com o bebê. Além da adaptação ao feto e ao bebê recémnascido, geralmente, a primeira gravidez traz implicações no relacionamento das mulheres com seus parceiros.

A literatura especializada, há décadas, vem divulgando pesquisas que mostram nos seus resultados relações entre papéis sexuais e relacionamento conjugal em geral (Belsky, Lang \& Huston, 1986; Deutsch \& Gilbert, 1976; Isaac \& Shah, 2004; Juni \& Grimm, 1993, 1994, 1994; MacDermid, Huston \& McHale, 1990; McGovern \& Meyers, 2002; Zammichieli, Gilroy \& Sherman, 1988). Também inúmeros dados que apóiam a idéia da associação entre papéis sexuais e saúde mental em geral têm sido apresentados (Bassoff, 1984; Bassoff \& Glass, 1982; Berthiaume, David, Saucier \& Borgeat, 1996; Cook, 1987; Harris \& Schwab, 1990; Williams \& D'Alessandro, 1994). No entanto, são escassas 
as pesquisas publicadas com sujeitos brasileiros que tenham verificado essas relações. Além disso, registra-se a publicação de alguns ensaios críticos, tais como Brasileiro, Jablonski e Féres-Carneiro (2002).

\section{Papéis Sexuais}

A revisão de Constantinople (1973), um marco na área, buscou definições teóricas e concluiu que masculinidade e feminilidade parecem estar entre os conceitos mais turvos do vocabulário dos psicólogos. A mais generalizada definição dos termos é que estes seriam traços relativamente duradouros que estariam mais ou menos enraizados na anatomia, fisiologia e experiências iniciais e, geralmente, serviriam para distinguir homens e mulheres na aparência, atitudes e comportamento.

Lenney (1991) observou que algumas abordagens teóricas definem papéis sexuais como as características, atitudes, valores e comportamentos que a sociedade especifica como apropriados para homens e mulheres. Gilbert (1985) sustentou que papéis sexuais se referem às expectativas normativas sobre a divisão de trabalho entre os sexos e as regras sobre interações sociais relativas ao gênero que existem numa cultura ou contexto histórico particular.

Stewart e McDermott (2004) comentaram que gênero é um fator ou variável empírica amplamente reconhecida na compreensão de vários aspectos do comportamento. Na psicologia, gênero é usado empiricamente, com freqüência, sem muita consciência de seu significado social ou conceitual. Dessa forma, os psicólogos usam-no de três maneiras completamente diferentes: para significar diferenças sexuais, variabilidade no sexo e gênero vinculado às relações de poder que estruturam muitas interações e instituições sociais.

Oliveira (1983) questionou por que mulheres e homens tendem a comportar-se de maneiras diversas. Até os anos 1960 , os pesquisadores sempre procuraram avaliar as características masculinas e femininas de personalidade baseados num modelo bipolar e unidimensional. Nessa perspectiva, o paradigma central é o pressuposto de que homens e mulheres diferem em muitas de suas características, nos estereótipos sociais, nos padrões de desempenho de papéis sexuais ou nas prescrições culturalmente aprovadas para indivíduos do sexo masculino e para indivíduos do sexo feminino: homens são mais independentes e agressivos e mulheres, mais dependentes e amáveis. Para o pesquisador, o sistema social parece guiar as crianças do sexo masculino a caminharem por um lado e as do sexo feminino por outro, na direção de como ser homem e como ser mulher, respectivamente. Oliveira (1983) comentou que nas últimas décadas muito tem sido escrito sobre as mudanças desses papéis sexuais, com homens apresentando emoções e mulheres sendo encorajadas a serem assertivas e negociadoras.

Na psicologia cognitiva convivem, principalmente, duas teorias cognitivas tradicionais que abordam o desenvolvimento do gênero: Kohlberg (1972) sustentou que o processo de tipificação se apóia no marco evolutivo geral da compreensão da realidade, afetando a organização cognitiva dos sexos sobre a qual se conformará gradativamente, a constância do gênero. Da interação entre os estereótipos de papéis sexuais e a suas identidades surgirão os seus atributos. Dessa forma, as mudanças na maturação cognitiva afetam a autopercepção e se refletem na categorização de estereótipos e valores sobre o sexo. Na Teoria de Processamento de Informação foram elaborados alguns modelos que explicam o desenvolvimento e funcionamento dos estereótipos de gênero com base em esquemas cognitivos integráveis no autoconceito. Partindo da idéia de que os sujeitos integram a informação recebida sobre uma base de esquemas previamente estabelecida, é lógico pensar que a designação social de gênero atuará prontamente, possibilitando o desenvolvimento de uma extensa rede de associações internas que, quando ativadas, serão decisivas para interpretar a realidade e, especialmente, o autoconceito (Barberá, 1998).

Para Sternberg (2000), conceito é a unidade essencial do conhecimento simbólico, a idéia que se tem sobre algum objeto. Na maioria dos casos, a idéia toda pode ser captada com um único termo, por exemplo, mulher. $\mathrm{O}$ conceito pode estar relacionado com muitos outros, no caso citado, mulher pode se relacionar com delicadeza, suavidade, afetividade, etc. Ou seja, os conceitos, em geral, se organizam nos esquemas. Os esquemas são estruturas mentais que representam o conhecimento e são constituídos por uma série de conceitos inter-relacionados organizados de forma significativa.

Na Gender Schema Theory (Bem, 1974, 1981), o esquema de gênero foi concebido como um contínuo simples, com dois pólos, em um dos quais estariam situados os sujeitos esquemáticos (fortemente masculinos ou femininos), enquanto, no extremo oposto, estariam os sujeitos com orientações fracas de papéis sexuais e os que apresentam tendências de sexo cruzado, não esquemáticos segundo o gênero ou não tipificados sexualmente. A classificação desses dois tipos (esquemáticos/tipificados e não esquemáticos/não tipificado) se baseou nas divergências quanto à disponibilidade cognitiva, que se manifestaria tanto em nível de discriminação perceptiva, associativa e de memória, quanto no referente às expectativas e crenças sobre a polaridade de gêneros. Os esquemáticos teriam maior predisposição para classificar as informações em categorias de masculino e feminino e para decidir que atributos incluiriam ou não em seu autoconceito.

O esquema cognitivo de gênero (Bem, 1981) estaria estreitamente ligado aos padrões sócioculturais de comportamentos esperados para cada um dos sexos. Uma vez aprendido, esse esquema predisporia a criança a perceber o mundo, também, em termos sexuais. Os esquemas de gênero serviriam para a criança avaliar a si própria e aos que a rodeiam em termos de adequação aos padrões definidos pela sociedade para os sexos, sentindo-se motivada a ajustar-se a essas definições. A auto-estima e o autoconceito seriam desenvolvidos, também, sob a regência do esquema de gênero.

As pessoas esquemáticas/tipificadas são aquelas que, em função de um "fator motivacional internalizado", organizam as informações em termos das definições culturais de masculinidade e feminilidade e cujo autoconceito e comportamento resultam desse processamento. Por outro lado, tais conotações não são marcantes para pessoas não esquemáticas ou não tipificadas. As diferenças individuais ficariam por conta do quanto, na história pessoal do indivíduo, foi enfatizada a importância das dicotomias de gênero (Bem, 1981). A androginia representaria uma forma particular de processar informação. Os andróginos, diferentes dos esquemáticos/tipificados, não contam com conotações sexuais para orientar o seu processamento de informação. Estes podem 
até estar inconscientes das diferenças quanto à propriedade ou impropriedade sexual de uma dada situação.

A noção de flexibilidade de papel sexual como uma característica dos andróginos tem sido proposta há mais de três décadas (Bem, 1974). Flexibilidade de papel sexual tem sido conceitualizada como uma qualidade adaptativa relativa à apresentação de comportamentos flexíveis às situações.

Cheng (2005) chegou à conclusão de que os andróginos podem não ter um conhecimento mais amplo de estratégias de coping do que os outros tipos de papéis sexuais, porém são mais flexíveis na distribuição de estratégias de coping conforme a necessidade de controle de diferentes estressores. A autora também verificou que andróginos experimentam níveis de depressão mais baixos em períodos estressantes do ciclo vital.

Diversas escalas têm sido desenvolvidas para medir papéis sexuais, sendo que as mais usadas são o Bem Sex Role Inventory (BSRI) de Bem (1974) e o Personal Attributes Questionnaire (PAQ) de Spence, Helmreich e Stapp (1975). Os resultados da pesquisa de Bem (1974) levaram às seguintes conclusões: 1) as dimensões da masculinidade e da feminilidade são empírica e logicamente independentes; 2) o conceito de androginia psicológica é fidedigno e está definido operacionalmente pela obtenção de índices altos ou baixos em ambas as escalas de masculinidade e feminilidade; 3 ) escores fortemente tipificados sexualmente não refletem uma tendência geral do indivíduo para responder em uma direção socialmente desejável, mas sim uma tendência específica para a autodescrição, em concordância com os padrões de comportamento desejáveis para homens e mulheres.

O BSRI foi revisado por Bem (1977) após as contribuições de Spence e cols. (1975). Estes argumentaram que as pessoas andróginas deveriam apresentar apenas índices elevados nas escalas de masculinidade e feminilidade. Pessoas com índices baixos deveriam ser classificadas como "indiferenciados".

A primeira adaptação do BSRI à cultura brasileira foi realizada por Oliveira (1983), uma reavaliação feita por Koller, Hutz, Vargas e Conti (1990) revelou diversos problemas com o instrumento que o levaram, em seguida, a uma readaptação (Hutz \& Koller, 1992). Hernandez (no prelo) verificou a estrutura desta readaptação e os resultados mostraram evidências de que a atual versão brasileira do instrumento continua sendo válida e fidedigna.

As duas maiores áreas ligadas à pesquisa em papéis sexuais são: ajustamento psicológico e relacionamento interpessoal. Coincidentemente, as duas grandes áreas de interesse na pesquisa em transição para a parentalidade têm sido a do ajustamento conjugal e emocional (Wilkinson, 1995). Os impulsos iniciais do trabalho de Bem (1975) apontaram para a idéia de que a maioria das pessoas bem ajustadas é andrógina. Isso contrasta com o modelo anterior de congruência, homens e mulheres típicos comporiam a maioria da população bem ajustada.

\section{Ajustamento Conjugal}

No presente trabalho, para mensurar o ajustamento conjugal, foi adotado o modelo (Spanier \& Cole, 1975) e o instrumento desenvolvido por Spanier (1976), que, em seu estudo de criação da Dyadic Adjustment Scale (DAS), considerou que o conceito de ajustamento conjugal tinha um lugar proeminente na pesquisa sobre o casamento e o relacionamento familiar até a metade dos anos 1970. Apesar disso, reconheceu a validade das críticas que classificavam o conceito de ajustamento conjugal como vago e ambíguo. Em decorrência, construiu um novo instrumento numa tentativa de melhorar a medida de ajustamento conjugal integrando definições nominais, operacionais e mensuração.

De acordo com Spanier (1976), o ajustamento conjugal pode ser visto em duas perspectivas distintas - como um processo ou uma avaliação qualitativa de um estado. Para o autor, definir ajustamento conjugal como um processo tem diversas implicações, sendo a mais importante delas a de que um processo pode ser melhor estudado ao longo do tempo. Sem dúvida, os estudos transversais na investigação do ajustamento têm algum valor, contudo, é evidente que um processo pode ser observado melhor mediante delineamentos longitudinais. Uma definição de processo é estabelecida não apenas pela existência de um continuum, mas também na crença de que há movimento junto com o continuum. Assim, o processo de ajustamento conjugal consiste nos eventos, circunstâncias e interações que movem o casal para frente e para trás junto com esse continuum.

A DAS teve sua primeira análise fatorial confirmatória realizada por Spanier e Thompson (1982), que encontraram uma solução para quatro fatores, explicando $94 \%$ da covariância entre os itens, confirmando a proposta original. Além disso, o coeficiente Alpha de Cronbach para a escala toda foi de 0, 91. Recentemente, Ahlborg, Persson e Hallberg (2005) avaliaram a DAS e fizeram modificações para uso com novos pais. Porém, esse novo instrumento foi publicado após o início da coleta de dados da investigação atual, o que impossibilitou o seu uso.

\section{Papéis sexuais e ajustamento conjugal}

Waldron e Routh (1981) estudaram casais esperando seus primeiros filhos. No pré-natal, não foi encontrada relação entre os papéis sexuais e o ajustamento conjugal. Destarte, nas últimas décadas, diversos estudos, em contextos variados (Antill, 1983; Baucom \& Aiken, 1984; Davidson \& Sollie, 1987; Juni \& Grimm, 1993, 1994; Lamke, 1989; Langis, Sabourin, Lussier \& Mathieu, 1994; Murstein \& Williams, 1983; Zammichieli \& cols., 1988), têm descoberto que o ajustamento conjugal está associado com papéis sexuais.

Os resultados de Antill (1983) indicaram que maridos e esposas com altos escores em feminilidade estavam mais satisfeitos com seus relacionamentos. Além disso, as mulheres estavam mais satisfeitas com homens tipificados femininos. Juni e Grimm (1993) encontraram escores de feminilidade correlacionados com satisfação conjugal mais do que os de masculinidade. Masculinidade foi mais correlacionada com satisfação conjugal para homens do que para mulheres. Juni e Grimm (1994), novamente, buscaram relação entre as categorias do BSRI e a satisfação conjugal. No entanto, em vez de abordar os sujeitos como indivíduos, examinaram os papéis sexuais do casal como um construto unitário. Os casais foram classificados como andróginos, congruentes, incongruentes e indiferenciados. Análises mostraram que casais andróginos retrataram um número maior de esposas que estavam insatisfeitas com respeito às questões envolvendo filhos. 
Para as mulheres, no estudo de Langis e cols. (1994), a satisfação conjugal foi associada com as qualidades femininas autopercebidas e com o nível ótimo de masculinidade e feminilidade percebidos em seus maridos. Além disso, pequenas discrepâncias medidas entre os níveis reais e ideais de masculinidade e feminilidade atribuídos aos parceiros constituíram-se em preditores confiáveis de satisfação conjugal.

Isaac e Shah (2004) não encontraram relação entre papéis sexuais e ajustamento conjugal para indivíduos indianos. Porém, análises para tipos de díades (diversas combinações de papéis sexuais dos casais) e ajustamento conjugal revelaram diferenças estatísticas significativas entre os grupos. As díades de casais andróginos estavam melhor ajustadas do que quaisquer outros tipos. As semitradicionais (quando um parceiro tinha papel sexual tradicional e o outro era andrógino ou indiferenciado, ou quando ambos eram indiferenciados) mostraram ajustamento conjugal mais pobre.

\section{Papéis sexuais e ajustamento emocional/neuroticismo}

Segundo Rodrigues, Pérez-López e Brito de la Nuez (2004), há escassa pesquisa que investigue casais durante a gravidez e relacione variáveis, tais como satisfação conjugal e outros aspectos da emocionalidade. Em geral, os trabalhos têm se concentrado em estudar os efeitos da chegada do bebê no período pós-parto. Contudo, para a maioria dos casais, essa transição começa na gravidez. Em torno de sete a $12 \%$ da população geral de mulheres grávidas têm apresentado taxas de depressão (Durkin, Morse \& Buist, 2001). Considerando que a depressão do pós-natal tem oscilado entre 10 a $15 \%$ da população de novas mães, não parece justo que o foco dos estudiosos no assunto esteja tão desequilibradamente alocado nesse período.

Na pesquisa de Bassoff (1984), os relatos indicaram que mulheres andróginas e tipificadas masculinas apresentaram escores menores em estresse psicológico do que as femininas ou indiferenciadas. Uma interação quase significativa entre masculinidade e feminilidade - com efeitos principais não significativos - na dimensão hostilidade de ajustamento deu apoio à noção de que novas mães andróginas são menos hostis do que as tipificadas masculinas, femininas ou indiferenciadas.

Os resultados de Hock, Schirtzinger, Lutz e Widaman (1995) indicaram que mães que relataram mais atitudes de papéis sexuais tradicionais expressaram mais sintomas depressivos durante a gravidez. Berthiaume e cols. (1996) não mostraram associação entre sintomatologia depressiva prénatal com papéis sexuais. Contudo, as medidas de bem-estar psicológico apresentaram relação com a androginia. A adoção de papel sexual andrógino, comparado com papel sexual indiferenciado, foi relacionada com maior satisfação diante do apoio recebido da rede social. Isso sugeriu que androginia está relacionada com características que promovem o desenvolvimento e manutenção de trocas interpessoais satisfatórias. A elevada auto-estima e o baixo nível de apreensão pré-natal foram, também, associados com mulheres andróginas.

A meta principal deste trabalho foi a verificação da relação entre os papéis sexuais e o ajustamento conjugal e emocional de mulheres grávidas de seus primeiros filhos e, nessa perspectiva, a associação da androginia com a qualidade do relacionamento interpessoal e com a saúde mental.
Além disso, foram verificadas as relações destas com outras variáveis coletadas, tais como sexo, idade, escolaridade, tempo de gestação, tempo de relação e tipo de gravidez (planejada ou não).

\section{Método}

\section{Participantes}

Foi constituída uma amostra de conveniência, de 135 mulheres primigestas. As participantes foram recrutadas entre os clientes dos setores de acompanhamento pré-natal (Ginecologia e Obstetrícia) dos seguintes locais: Hospital de Clínicas de Porto Alegre/UFRGS, Hospital de Gravataí/RS, Hospital Presidente Vargas, Hospital Fêmina e Posto de Saúde Santa Marta do município de Porto Alegre/RS, clientes do Sistema Único de Saúde (SUS). Além disso, alguns sujeitos foram abordados em grupos de gestantes no Centro Espírita Bezerra de Menezes (POA/RS). As participantes deviam coabitar com seus parceiros, caracterizando a vida conjugal, mas não precisavam ser formalmente casadas.

A Idade média das mulheres foi de 23,9 anos $(D P=6,83)$, o tempo de relação médio foi de 45,2 meses $(D P=41,90)$ e o tempo de gestação médio foi cinco meses $(D P=0,50)$. A escolaridade ficou distribuída da seguinte maneira: ensino fundamental incompleto 28 (20,7\%); ensino fundamental completo nove (6,7\%); ensino médio incompleto $15(11,1 \%)$; ensino médio completo 31 (23\%); ensino superior incompleto 17 (12,6\%); ensino superior completo $15(11,1 \%)$; e não forneceram esse dado 20 (14,8\%). Quanto ao tipo de gravidez, as participantes declararam 57 ocorrências de gravidez planejada (42,2\%) e 58 de gravidez acidental (43\%), 20 omitiram esse dado (14,8\%).

\section{Instrumentos}

1) O Bem Sex Role Inventory (BSRI), de Bem (1974), adaptado por Oliveira (1983), readaptado por Hutz e Koller (1992) e reavaliado por Hernandez (no prelo).

Esse instrumento de avaliação de Papéis Sexuais é um conjunto de 60 itens de adjetivos que compõem três escalas: masculina, feminina e neutra. Os respondentes utilizaram uma escala tipo Likert de sete pontos (1, que significa que a característica nunca é verdadeira, até 7 , que significa que a característica é sempre verdadeira) para avaliar a autopercepção das participantes.

Para determinar o ponto de corte e classificar os indivíduos nos papéis sexuais, foi utilizado o cálculo da mediana das médias dos escores dos sujeitos em cada uma das escalas (Bem, 1977). Os pontos de corte calculados foram: 5,55 na escala feminina e 4,25 na escala masculina. As participantes que apresentaram escores acima do ponto de corte na escala masculina e abaixo do ponto de corte na escala feminina foram classificadas na categoria tipificadas masculina; escores acima do ponto de corte na escala feminina e abaixo do ponto de corte na escala masculina foram classificados na categoria tipificada feminina; acima do ponto de corte em ambas as escalas, masculina e feminina, foram classificadas na categoria andrógino; escores situados abaixo do ponto de corte em ambas as escalas, masculina e feminina, foram classificadas na categoria indiferenciado. 
Os Alphas de Cronbach, calculados por Hernandez (no prelo) para a versão brasileira do BSRI, foram 0,90 para a escala feminina, 0,81 para a escala masculina e 0,61 para a escala neutra.

2) A Dyadic Adjustment Scale (DAS), criada por Spanier (1976) e, recentemente, verificada por Hernandez (no prelo), é uma escala de 32 itens que buscam medir as seguintes dimensões:

Consenso diádico: avalia a percepção do nível de concordância do casal sobre uma variedade de questões básicas da relação, tais como: financeiras, lazer, religiosas, amizades, convencionalidade, filosofia de vida, negócios com parentes, metas, tempo gasto junto, tomadas de decisão, tarefas domésticas, tempo com lazer e decisões profissionais.

Satisfação diádica: mede a percepção das questões relativas à discussão do divórcio, à saída de casa após briga, ao arrependimento com o casamento, às querelas, à implicância mútua, ao estar bem, à confiança no cônjuge, ao beijo no cônjuge, ao grau de felicidade e ao compromisso com o futuro do relacionamento.

Coesão diádica: examina o senso de compartilhamento emocional do casal. Esses itens medem a percepção relativa ao engajamento mútuo em interesses externos, à estimulação de idéias, à diversão conjunta, à discussão tranqüila e ao trabalho conjunto em projetos.

Expressão diádica de afeto: mede a percepção da concordância dos membros do casal sobre as demonstrações de afeto, as relações sexuais, a falta de amor e as recusas ao sexo.

A DAS é um instrumento que utiliza escalas tipo Likert para aferir ajustamento diádico ou conjugal. Em alguns itens são usadas escalas de cinco pontos, em outros, seis pontos e até sete pontos, em geral, os pontos extremos das escalas significando "nunca" e "todo o tempo", respectivamente. Além disso, os itens 29 e 30 apresentam apenas duas opções, "sim" ou "não". Para aumentar a fidedignidade da escala, alguns itens são afirmações positivas, outros são afirmações negativas. Portanto, algumas respostas requerem reversão de escores antes da apuração da pontuação.

O escore total da escala pode variar de 0 a 151 , e é obtido pela soma total dos escores nas quatro subescalas. De acordo com Spanier (1976), os indivíduos que obtiverem 101 pontos ou menos devem ser classificados como na vivência de um relacionamento de sofrimento ou desajustado e os sujeitos que alcançarem 102 pontos ou mais estariam vivenciando um relacionamento sem sofrimento ou bem ajustado.

Os Alphas de Cronbach, calculados por Hernandez (no prelo) para a versão brasileira da DAS, foram: 0,86 para a subescala consenso diádico, 0,86 para a subescala satisfação diádica, 0,76 para a subescala coesão diádica e 0,62 para a subescala expressão de afeto.

3) A Escala Fatorial de Neuroticismo, de Hutz e Nunes (2001): Esse instrumento avalia uma dimensão de personalidade chamada de ajustamento emocional ou neuroticismo inspirado no modelo dos Cinco Grandes Fatores. A escala possui 82 itens (frases afirmativas) que atendem a quatro fatores: vulnerabilidade, que avalia a intensidade com que as pessoas experimentam sofrimento como conseqüência da aceitação dos outros; desajustamento psicossocial, que identifica pessoas que tendem a ser muito agressivas e hostis com os outros, mentindo e manipulando a situação em proveito próprio; ansiedade, que identifica pessoas que tendem a ser emocionalmente instáveis; e depressão, que avalia os padrões usados pelas pessoas para interpretar os eventos que ocorrem em suas vidas.

Todos os itens são medidos por uma escala tipo Likert de sete pontos (1 significando afirmação completamente inadequada e 7 significando afirmação perfeitamente adequada). Os autores (Hutz \& Nunes, 2001) advertem que alguns itens precisam ter seus escores invertidos para obtenção dos escores totais. Além disso, comentam que a maior parte da população deve obter entre 80 e 120 escores padronizados na EFN e que ainda não foi possível determinar um ponto de corte que discrimine normalidade e patologia. Contudo, escores padronizados muito elevados ou muito baixos, possivelmente devem indicar algum tipo de transtorno de personalidade.

Os Alphas de Cronbach encontrados por Hutz e Nunes (2001) para a EFN total foram: 0,94 para a escala de vulnerabilidade, 0,89 para a escala desajustamento psicossocial, 0,82 para a escala ansiedade, 0,87 para a escala depressão.

\section{Procedimentos}

Os participantes foram abordados individualmente no momento da consulta pré-natal nas instituições de saúde anteriormente citadas e convidados a participar da pesquisa. Sempre, no início da abordagem, era feito um breve rapport, informando sobre a pesquisa e solicitando o preenchimento e assinatura de um Termo de Consentimento Livre e Informado. Geralmente, os questionários eram preenchidos nas salas de espera dos hospitais e postos de saúde. Além dos instrumentos, eram fornecidas aos participantes uma prancheta e uma caneta. Algumas vezes, as respostas foram dadas em etapas, antes e depois da consulta e, até, em dias subseqüentes. Houve também a ocorrência de serem respondidos durante curso de preparação de grávidas, nesse caso, diversas gestantes preencheram simultaneamente os instrumentos. No Centro Espírita, os membros dos casais, isolados, responderam aos questionários em uma sala cedida para esse fim. Em alguns casos, os dados foram coletados nas residências dos sujeitos mediante anuência e acerto prévio com os mesmos.

\section{Delineamento e análise dos dados}

Foi realizada uma pesquisa transversal do tipo correlacional. Foram verificadas as relações entre os papéis sexuais e os ajustamentos conjugal e emocional. Os escores de cada sujeito foram digitados e processados pelo SPSS, versão 11.5, utilizando estatística descritiva, análise de variância e regressão múltipla.

\section{Resultados}

Do total de 135 participantes, 34 não responderam ao BSRI e, portanto, não puderam ser classificadas quanto aos papéis sexuais. Foi executada a classificação de papéis sexuais de 101 mulheres: 19 tipificadas femininas (18,8\%), 18 tipificadas masculinas $(17,8 \%), 29$ andróginas $(28,7 \%)$ e 35 indiferenciadas $(34,7 \%)$.

Conforme pode ser observado na Tabela 1, ANOVA para o fator papéis sexuais e ajustamento conjugal revelou diferenças estatísticas significativas para as médias da DAS total e da subescala consenso diádico entre os grupos. O teste de post hoc de Scheffé identificou, em ambos os casos, que a média dos 
Tabela 1. Médias, desvios padrão e ANOVA de papéis sexuais por ajustamento conjugal.

\begin{tabular}{|c|c|c|c|c|c|c|c|}
\hline Subescalas & Papéis Sexuais & $n$ & $M$ & $D P$ & Erro Padrão & $F$ & $p<$ \\
\hline \multirow[t]{4}{*}{ Consenso } & Típico Feminino & $17^{*}$ & 3,4 & 47 & 11 & 3.570 & 0,01 \\
\hline & Típico Masculino & 18 & 3,5 &, 86 & ,19 & & \\
\hline & Andrógino & 29 & 3,8 & 65 &, 11 & & \\
\hline & Indiferenciado & 34 & 3,2 & ,92 &, 15 & & \\
\hline \multirow[t]{4}{*}{ Satisfação } & Típico Feminino & 17 & 3,8 & 41 & ,09 & 1.397 & ns \\
\hline & Típico Masculino & 18 & 3,6 & ,79 &, 17 & & \\
\hline & Andrógino & 29 & 3,9 &, 52 & ,09 & & \\
\hline & Indiferenciado & 34 & 3,6 & ,68 &, 11 & & \\
\hline \multirow[t]{4}{*}{ Coesão } & Típico Feminino & 17 & 3,2 & 81 & 18 & 1.592 & ns \\
\hline & Típico Masculino & 18 & 3,4 &, 80 &, 18 & & \\
\hline & Andrógino & 29 & 3,7 &, 77 &, 13 & & \\
\hline & Indiferenciado & 34 & 3,3 &, 72 &, 12 & & \\
\hline \multirow[t]{4}{*}{ Expressão de Afeto } & Típico Feminino & 17 & 2,4 &, 52 &, 11 & 253 & ns \\
\hline & Típico Masculino & 18 & 2,4 & ,43 & ,09 & & \\
\hline & Andrógino & 29 & 2,4 &, 47 & 08 & & \\
\hline & Indiferenciado & 34 & 2,3 & ,62 &, 10 & & \\
\hline \multirow[t]{4}{*}{ DAS Total } & Típico Feminino & 17 & 3,4 & ,30 & ,07 & 2.837 & 0,04 \\
\hline & Típico Masculino & 18 & 3,4 & 67 &, 15 & & \\
\hline & Andrógino & 29 & 3,7 &, 50 & ,09 & & \\
\hline & Indiferenciado & $34^{\mathrm{a}}$ & 3,2 & ,65 &, 10 & & \\
\hline
\end{tabular}

"Dois indivíduos classificados como típicos femininos e um indiferenciado não responderam a DAS.

escores do grupo dos andróginos foi significativamente maior do que a dos indiferenciados. Não foi identificada diferença estatística significativa entre as médias dos outros tipos de papéis sexuais. Para as outras subescalas da DAS (satisfação diádica, coesão diádica e expressão de afeto), também não foram apuradas diferenças estatísticas significativas.

ANOVA para o fator papéis sexuais e ajustamento emocional não apresentou diferenças estatísticas significativas $(p<0,05)$ entre as médias dos grupos.

Foi executada uma série de análises de regressão, método Stepwise, para as variáveis dependentes DAS total e suas subescalas (consenso diádico, satisfação diádica, coesão diádica e expressão de afeto), tendo como variáveis independentes sexo, idade, tempo de relação, tipo de gravidez, tempo de gestação, escalas feminina e masculina do BSRI e a EFN total e suas escalas (vulnerabilidade, desajustamento psicossocial, ansiedade e depressão).

Para a variável dependente consenso foram ajustados cinco modelos que explicaram $28 \%$ da variância. A melhor preditora de consenso foi a variável independente ansiedade medida pela EFN, responsável por $14 \%$ da variância explicada. As variáveis independentes tempo de gestação, masculinidade, feminilidade e sexo tiveram uma participação menor.

Para a variável dependente satisfação foram ajustados quatro modelos que explicaram $31 \%$ da variância. A melhor preditora foi a variável independente ajustamento emocional medida pela EFN total, com $21 \%$ da variância explicada. As variáveis independentes vulnerabilidade, sexo e feminilidade tiveram uma participação menor.

Para a variável dependente coesão foram ajustados três modelos que explicaram $9 \%$ da variância. A melhor preditora foi a variável independente depressão medida pela EFN, com $5 \%$ da variância explicada. As variáveis independentes idade e masculinidade tiveram uma participação menor.
Para a variável dependente expressão de afeto foi ajustado um único modelo que explicou $6 \%$ da variância. A única variável independente preditora foi a escala depressão medida pela EFN.

Para a variável dependente DAS total foram ajustados quatro modelos que explicaram $30 \%$ da variância. A melhor preditora foi a variável independente EFN total, com $18 \%$ da variância explicada. As variáveis independentes vulnerabilidade, sexo e feminilidade tiveram uma participação menor.

\section{Discussão}

Na comparação dos grupos de papéis sexuais, os resultados revelaram diferenças estatísticas significativas entre as médias dos andróginos e indiferenciados obtidas na DAS total e na subescala consenso. Nos dois casos, os andróginos obtiveram escores médios superiores aos dos indiferenciados em ajustamento conjugal durante a gravidez do primeiro filho. Esse resultado já está razoavelmente validado nessa linha de pesquisa. Pesquisadores (Antill, 1983; Baucom \& Aiken, 1984; Davidson \& Sollie, 1987; Isaac \& Shah, 2004; Juni \& Grimm,1994; Kurdek \& Schmitt, 1986; Lamke, 1989; Murstein \& Williams, 1983; Zammichieli \& cols., 1988), em variados contextos, têm encontrado escores mais elevados em ajustamento conjugal para os andróginos do que para outros tipos de papéis. Ao mesmo tempo, estudos, tais como Davidson e Sollie (1987), mostraram que indivíduos classificados como indiferenciados são mais dispostos ao desajustamento.

Para grande parte das mulheres, a etapa da gravidez na transição para a parentalidade é um período de dramáticas mudanças biológicas, psicológicas e sociais. Como uma conseqüência dessas mudanças e processos de adaptação, esse período é, com freqüência, associado com aumento da sensibilidade, labilidade e ansiedade (Öhman \& cols., 2003). 
Durkin e cols. (2001) mostraram que o estresse, vinculado ao papel de gênero, teve uma forte associação com as emoções durante a gravidez média. Tornar-se pais produz um aumento das diferenças biológicas entre homens e mulheres e aumenta a consciência dos papéis de gênero tradicionais associados com a parentalidade. Os "casais grávidos" podem sentir pressão para se conformar às convenções devido às auto-expectativas, às expectativas dos parentes, dos pares e das normas sociais. Isso pode envolver uma ampla modificação na autopercepção, capaz de resultar em estresse e baixa do humor em certos indivíduos mais vulneráveis e refletir no ajustamento conjugal.

Por meio da análise de regressão, pode-se constatar que a ansiedade medida pela EFN foi responsável pela maior parte da variância do consenso. Ainda, reforçando a relação dos papéis sexuais com o consenso, os escores das escalas masculinas e femininas do BSRI também explicaram uma parte menor da variância. Esses dados revelam que a interação ansiedade medida pela EFN e as características vinculadas aos sexos (escalas do BSRI) estiveram consideravelmente associadas ao consenso e, conseqüentemente, sugerindo que as grávidas experimentavam um ambiente, pelo menos em parte, semelhante ao descrito antes (Durkin \& cols., 2001; Öhman \& cols., 2003).

Berthiaume e cols. (1996) relataram que os andróginos, comparados com os indiferenciados, mostraram-se significativamente mais satisfeitos com o apoio fornecido pela rede social durante a gravidez. Os autores explicaram que a androginia está relacionada com características disposicionais que promovem o desenvolvimento e manutenção de trocas interpessoais mais satisfatórias.

As pessoas andróginas têm sido relatadas como sendo mais adaptativas (Bem, 1975; Bem, Martyna \& Watson, 1976) e mais flexíveis (Spence \& cols., 1975; Cheng, 2005). $\mathrm{O}$ ajustamento conjugal durante a gravidez do primeiro filho requer um grau significativo de adaptação e flexibilidade. Para ser uma pessoa bem ajustada à conjugalidade, pode ser necessário possuir características instrumentais tais como assertividade e disposição para o risco e, também, características expressivas tais como sensibilidade e compreensão. Os andróginos têm atributos instrumentais e expressivos e, por isso, é compreensível que tenham um ajustamento conjugal significativamente melhor do que os indiferenciados.

A noção de flexibilidade de papel sexual, como uma característica dos andróginos, tem sido proposta há mais de três décadas (Bem, 1974). A flexibilidade de papel sexual tem sido definida como uma qualidade adaptativa relativa à apresentação de comportamentos flexíveis às situações. Cheng (2005) examinou a flexibilidade dos papéis sexuais durante uma variedade de eventos estressantes semelhantes à gravidez. Os andróginos demonstraram mais sensibilidade para discriminar sutis diferenças entre os diversos eventos, como pode ser notado nas habilidades para distribuir diferentes estratégias situacionais efetivas de coping. O papel chave da flexibilidade - "pensamento prático hábil" - é estimular uma integração estável da personalidade e das características do ambiente de forma adaptativa.

Os níveis mais baixos de ajustamento conjugal dos indiferenciados sugerem que essas mulheres têm um número limitado de papéis e habilidades de adaptação, apresentando mais dificuldades para lidar com as inúmeras demandas situacionais da conjugalidade no contexto de mudanças, como, por exemplo, com a demanda de ansiedade identificada neste caso.

\section{Conclusão}

Houve uma confirmação parcial das expectativas neste estudo. A tese de que os andróginos apresentariam melhor desempenho no relacionamento interpessoal foi totalmente apoiada. No entanto, no aspecto saúde mental, a esperada supremacia desses andróginos não foi confirmada pelos dados.

Sugere-se que novas investidas de pesquisa possam ser realizadas, incluindo a participação do sexo masculino. Mais do que isso, que novas pesquisas escolham o casal como a unidade investigada, considerando as possibilidades de combinações de papéis sexuais dos cônjuges e suas relações com o ajustamento conjugal e emocional.

Os dados aqui produzidos poderão, posteriormente, contribuir no trabalho de promoção da saúde de mulheres primigestas/primíparas na atividade psicoprofilática em programas pré-natais com casais e, também, na clínica psicológica da saúde da família. Muitos pesquisadores imbuídos da educação de casais têm se dedicado a fazer essa ponte entre a pesquisa e a intervenção psicológica (Brotherson, 2004; Halford, Markman, Kline \& Stanley, 2003).

Cowan e Cowan (1995) mostraram a importância dos programas para facilitar a transição de casais para a parentalidade. Eles realizaram um estudo controlado de intervenção conjugal preventiva e demonstraram o efeito da terapia grupal de apoio durante a gravidez, reduzindo a queda da satisfação matrimonial e, dessa forma, prevenindo o divórcio e melhorando a qualidade da parentalidade.

Fica a sugestão ao psicólogo da saúde na execução dos programas de prevenção para valorizar os papéis sexuais como um aspecto significativo na conceitualização do ajustamento de casais durante a gravidez.

\section{Referências}

Ahlborg, T., Persson, L-O. \& Hallberg, L. R-M. (2005). Assessing the quality of the dyadic relationship in first-time parents: Development of a new instrument. Journal of Family Nursing, 11, 19-37.

Antill, J. K. (1983). Sex role complementarity versus similarity in married couples. Journal of Personality and Social Psychology, 45, 145-155.

Barberá, E. (1998). Psicología del Género. Barcelona: Ariel.

Bassoff, E. S. (1984). Relationships of sex-role characteristics and psychological adjustment in new mothers. Journal of Marriage and the Family, 46, 449-454.

Bassoff, E. S. \& Glass, G. V. (1982). The relationship between sex roles and mental health: a meta-analysis of twenty-six studies. The Counseling Psychologist, 10, 105-112.

Baucom, D. H. \& Aiken, P. A. (1984). Sex role identity, marital satisfaction, and response to behavioral marital therapy. Journal of Consulting and Clinical Psychology, 52, 155-162.

Belsky, J., Lang, M. \& Huston, T. L. (1986). Sex typing and division of labor as determinants of marital change across the transition to parenthood. Journal of Personality and Social Psychology, 50, 517-522.

Bem, S. L. (1974). The measurement of psychological androgyny. Journal of Consulting and Clinical Psychology, 42, 155-162. 
Bem, S. L. (1975). Sex role adaptability: One consequence of psychological androgyny. Journal of Personality and Social Psychology, 31, 634-643.

Bem, S. L. (1977). On the utility of alternative procedures for assessing psychological androgyny. Journal of Consulting and Clinical Psychology, 45, 196-205.

Bem, S. L. (1981). Gender Schema Theory: a cognitive account of sex typing. Psychological Review, 88, 354-364.

Bem, S. L., Martyna, W. \& Watson, C. (1976). Further explorations of the expressive domain. Journal of Personality and Social Psychology, 34, 1016-1023.

Berthiaume, M., David, H., Saucier, J. F. \& Borgeat, F. (1996). Correlates of gender role orientation during pregnancy and the postpartum. Sex Roles: A Journal of Research, 35, 781-800.

Brasileiro, R. de F., Jablonski, B. \& Féres-Carneiro, T. (2002). Papéis de gênero, transição para a paternalidade e a questão da tradicionalização. Psico, 33(2), 289-310.

Brotherson, S. (2004). Preparing for parenthood (On-line). NDSU Extension Service. North Dakota State University. Retirado em 30/05/2005 do site http://www.ext.nodak.edu/extpubs/yf/famsci/ fs601w.htm

Carter, E. \& McGoldrick, M. (2001). As mudanças no ciclo de vida familiar. Porto Alegre: ArtMed.

Cheng, C. (2005). Processes underlying gender-role flexibility do androgynous individuals know more or know how to cope? Journal of Personality, 73, 645-673.

Constantinople, A. (1973). Masculinity-femininity: An exception to a famous dictum? Psychological Bulletin, 80, 389-407.

Cook, E. P. (1987). Psychological androgyny: A review of the research. The Counseling Psychologist, 15, 471-513.

Cowan, C. P. \& Cowan, P. A. (1995). Interventions to ease the transition to parenthood: Why they are needed and what they can do. Family Relations, 44(4), 412-424.

Davidson, B. \& Sollie, D. L. (1987). Sex role orientation and marital adjustment. Social Behavior and Personality, 15, 59-69.

Deutsch, C. J. \& Gilbert, L. A. (1976). Sex role stereotypes: effect on perceptions of self and others on personal adjustment. Journal of Counseling Psychology, 23, 373-379.

Durkin, S., Morse, C. \& Buist, A. (2001). The factor structure of prenatal psychological and psychosocial functioning in firsttime expectant parents. Journal of Reproductive and Infant Psychology, 19, 121-134.

Gilbert, L. A. (1985). Measures of psychological masculinity and femininity: A comment on Gaddy, Glass, and Arnkoff. Journal of Couseling Psychology, 32, 163-166.

Goldberg, W. A., Michaels, G. Y. \& Lamb, M. E. (1985). Husbands' and wives' adjustment to pregnancy and first parenthood. Journal of Family Issues, 6(4), 483-503.

Halford, W. K., Markman, H. J., Kline, G. H. \& Stanley, S. M. (2003). Best practice in couple relationship education. Journal of Marital and Family Therapy, 29, 385-406.

Harris, T. L. \& Schwab, R. (1990). Sex-role orientation and personal adjustment. Journal of Social Behavior and Personality, 5, 473-479.

Hernandez, J. A. E. (no prelo). Reavaliando o Bem Sex Role Inventory. Estudos em Psicologia.

Hernandez, J. A. E. (no prelo). Avaliação estrutural da Escala de Ajustamento Diádico. Psicologia em Estudo.

Hock, E., Schirtzinger, M. B., Lutz, M. B. \& Widaman, K. (1995). Maternal depressive symptomatology over the transition to parenthood: Assessing the influence of marital satisfaction and marital sex role traditionalism. Journal of Family Psychology, 9(1), 79-88.

Hutz, C. \& Koller, S. (1992). A mensuração do gênero: Uma readaptação do BSRI. Psicologia: Reflexão e Crítica, 5, 15-21.

Hutz, C. S. \& Nunes, C. H. S. S. (2001). Escala Fatorial de Ajustamento, Emocional/Neuroticismo. São Paulo: Casa do Psicólogo.

Isaac, R. \& Shah, A. (2004). Sex roles and marital adjustment in Indian couples. International Journal of Social Psychiatry, 50, 129-141.

Juni, S. \& Grimm, D. W. (1993). Marital satisfaction and sex-roles in a New York metropolitan sample. Psychological Reports, 73, 307-314.

Juni, S. \& Grimm, D. W. (1994). Marital satisfaction as a function of dyadic gender-role constellations. The American Journal of Family Therapy, 22, 106-112.

Knauth, D. G. (2001). Marital change during the transition to parenthood. Pediatric Nursing, 27, 169-184.

Kohlberg, L. (1972). Análisis de los conceptos y actitudes infantiles relativos al papel.sexual desde el punto de vista del desarrollo cognitivo. Em E. E. Maccoby (Org.), Desarrollo de las diferencias sexuales (pp. 61-147). Madrid: Ediciones Marova.

Koller, S., Hutz, C., Vargas, S. \& Conti, L. (1990). Mensuração de gênero: Reavaliação do BSRI. Ciência e Cultura, 42, 517-518.

Kurdek, L. A. \& Schmidt, J. (1986). Interaction of sex-role selfconcept with relationship quality and relationship beliefs in married, heterossexual, cohabitating, gay, and lesbian couples. Journal of Personality and Social Psychology, 51, 365-370.

Langis, J., Sabourin, S., Lussier, Y. \& Mathieu, M. (1994). Masculinity, femininity, and marital satisfaction: An examination of theoretical models. Journal of Personality, 63, 393-414.

Lamke, L. (1989). Marital adjustment among rural couples: The role of expressiveness. Sex Roles, 21, 579-590.

Lenney,E. (1991). Sex Roles: The measurement of masculinity, femininity, and androgyny. Em J. P. Robinson, P. R. Shaver \& L. S. Wrightman (Orgs.), Measures of Personality and Social Psychological Attitudes (pp. 573-660). San Diego: Academic Press.

MacDermid, S. M., Huston, T. L. \& McHale, S. M. (1990). Changes in marriage associated with the transition to parenthood: Individual differences as a function of sex-role attitudes and changes in the division of household labor. Journal of Marriage and the Family, 52, 475-486.

McGovern, J. M. \& Meyers, S. A. (2002). Relationships between sex-role attitudes, division of household tasks, and marital adjustment. Contemporary Family Therapy, 24, 601-618.

Murstein, B. \& Williams, P. (1983). Sex roles and marital adjustment. Small Group Behavior, 14, 77-94.

Öhman, S. G., Grunewald, C. \& Waldenström, U. (2003). Women's worries during pregnancy: Testing the Cambridge Worry Scale on 200 Swedish women. Scandinavian Journal of Caring Sciences, 17, 148-152.

Oliveira, L. S. O. (1983). Masculinidade feminilidade androginia. Rio de Janeiro: Achiamé.

Rodrigues, A. R., Pérez-López, J. \& Brito de la Nuez, A. G. (2004). La vinculación afectiva prenatal y la ansiedad durante los últimos tres meses del embarazo en las madres y padres tempranos. Anales de Psicología, 20, 95-102.

Spanier, G. B. (1976). Measuring dyadic adjustment: New scales for assessing the quality of marriage and similar dyads. Journal of Marriage and the Family, 38, 15-28. 
Spanier, G. B. \& Cole, C. L. (1975). Toward clarification and investigation of marital adjustment. International Journal of Sociology of the Family, 6, 121-146.

Spanier, G. B. \& Thompson, L. (1982). A confirmatory analysis of the Dyadic Adjustment Scale. Journal of Marriage and the Family, 44, 731-738.

Spence, J. T., Helmreich, R. \& Stapp, J. (1975). Ratings of self and peers on sex role attributes and their relation to self-esteem and conceptions of masculinity and femininity. Journal of Personality and Social Psychology, 32, 29-39.

Sternberg, R. J. (2000). Psicologia cognitiva. Porto Alegre: ArtMed.

Stewart, A. J. \& McDermott, C. (2004). Gender in psychology. Annual Review of Psychology, 55, 519-544.

Waldron, H. \& Routh, D. K. (1981). The effect of the first child on the marital relationship. Journal of Marriage and the Family, 43, 785-788.
Wilkinson, R. B. (1995). Changes in psychological health and the marital relationship through childbearing: Transition or process as stressor? Australian Journal of Psychology, 47, 86-92.

Williams, D. E. \& D'Alessandro, J. D. (1994). A comparison of three measures of androgyny and their relationship to psychological adjustment. Journal of Social Behavior and Personality, 9, 469-480.

Zammichieli, M. E., Gilroy, F. D. \& Sherman, M. F. (1988). Relation between sex-role orientation and marital satisfaction. Personality and Social Psychology Bulletin, 14, 747-754.

Recebido em 18.01.2006

Primeira decisão editorial em 20.04.2006

Versão final em 20.05.2006

Aceito em 10.02.2007 